\title{
Outlet Control, Pathogenesis, and Treatment: The Role of Ultrasound in Its Management
}

\author{
${ }^{1}$ Abdel KM El Hemaly, ${ }^{2}$ Laila AES Mousa, ${ }^{3}$ Asim Kurjak, ${ }^{4}$ Ibrahim M Kandil, ${ }^{5}$ Abdel AA Aldarwish \\ ${ }^{6}$ Muhammad R Morad, ${ }^{7}$ Mervat M Ibrhaim, ${ }^{8}$ Mahmoud Eledaisy, ${ }^{9}$ Khaled Shehata, ${ }^{10}$ Mona M Ragab
}

\begin{abstract}
Introduction: Outlet control means continence, which is how to control body excreta (urine, flatus, and feces), control of temperance, body reaction, and control of sexual behavior and premature ejaculation. It is a nerve-muscle action, controlled by the central nervous system (CNS). Outlet control is an acquired behavior gained by learning and training to control the sympathetic nervous system. Although the sympathetic nervous system is part of the involuntary autonomic nervous system control, its function may be controlled. Evidence of this is seen in the control of body excreta control after toilet training and in how domestic animals can be trained to control body excreta as well.
\end{abstract}

Micturition and urinary continence: Urinary continence depends on a closed and empty urethra created by two factors: one is the presence of a strong intact internal urethral sphincter (IUS), which is a collagen-muscle tissue cylinder that extends from the bladder neck down to the perineal membrane. The other factor is an acquired behavior which is keeping high sympathetic tone at the IUS gained in early childhood from toilet training. Failure of either factors leads to urinary incontinence (UI).

Defecation and fecal continence: Fecal continence depends on a closed and empty anal canal created by two factors, one is inherent and one is acquired. The acquired factor is keeping high sympathetic tone at the internal anal sphincter (IAS) gained in early childhood from toilet training. The inherent factor is the presence of an intact strong IAS, which is a collagen-muscle tissue cylinder surrounding the anal canal.

Pathology: Childbirth trauma (CBT) causes laceration in the collagen chassis of the vagina leading to vaginal prolapse, and the intimately related IUS in front and/or the IAS posterior, causing $\mathrm{UI}$ and/or fecal incontinence (FI).

Pathophysiology: Outlet control is how to control the sympathetic nervous system and to manage different responses according to social circumstances.

\footnotetext{
${ }^{1-8}$ Professor, ${ }^{9}$ Lecturer, ${ }^{10}$ Consultant

${ }^{1,4-8}$ Department of Obstetrics and Gynecology, Al Azhar University, Cairo, Egypt

2Department of Pathology, Al Azhar University, Cairo, Egypt

${ }^{3}$ Department of Obstetrics and Gynecology, University of Zagreb Zagreb, Croatia

${ }^{9}$ Department of Radiology, Ain Shams University, Cairo, Egypt

${ }^{10}$ Department of Obstetrics and Gynecology, Al-Galaa Maternity Hospital, Cairo, Egypt
}

Corresponding Author: Abdel KM El Hemaly, Professor Department of Obstetrics and Gynecology, Faculty of Medicine Al Azhar University, Cairo, Egypt, Phone: +201001577969 e-mail: profakhemaly@hotmail.com
Diagnosis: Structural damage is diagnosed clinically and by medical imaging: ultrasound (US), magnetic resonance imaging (MRI) and/or computed tomography (CT) scan. Structural damage will cause functional disturbance which is assessed clinically and by urodynamics.

Conclusion: Ultrasound scanning of the urethra to see if it is closed or open and the state of the IUS and visualizing the lacerations are easy and cheap methods of assessment of UI. Also US assessment of the anal canal, open or closed, and the IAS, and the extent of its laceration will help very much in its diagnosis and management.

Keywords: 3DUS, Collagen chassis, Micturition and urinary continence, Outlet control, Urinary incontinence.

How to cite this article: El Hemaly AKM, Mousa LAES, KurjakA, Kandil IM, Aldarwish AAA, Morad MR, Ibrhaim MM, Eledaisy M, Shehata K, Ragab MM. Outlet Control, Pathogenesis, and Treatment: The Role of Ultrasound in Its Management. Donald School J Ultrasound Obstet Gynecol 2018;12(1):4-12.

Source of support: Nil

Conflict of interest: None

\section{INTRODUCTION}

Every body organ has a strong collagen chassis which is responsible for its shape, its integrity, and its location in the body. Lacerations or damage of the collagen chassis will distort the shape, site, and functions of the affected organ. Anybody action is a nerve-muscle action controlled by healthy and alert CNS. The nerve produces neurotransmitter which acts on neuroreceptors on the target organ leading to a response action. The sympathetic nervous system (thoracolumbar) has its postganglion nerve fibers secrete norepinephrine as its neurotransmitter. Its main effect is "fight or flight."

We introduced two other response actions which are gained by CNS: sympathetic training and control. The first of these is the fight-flight response to aggressive actions and staying calm and continent. However, if the stimulus is overwhelming, sympathetic "failure and incontinence" is the result. ${ }^{1,2}$

In a previous study, we proved the presence of high alpha-sympathetic tone at the IUS by urodynamic studies. We recorded in adult women the urethral pressure profile, then we gave $5 \mathrm{mg}$ intravenously alphasympathetic blocker drug, e.g., phentolamine (Regitine, Ciba-Geigy, Basle, Switzerland). The urethral pressure dropped significantly. Then, by intravenous drip, we injected alpha-sympathomimetic drug, norepinephrine 
(Levophed, Sanofi Winthrop, Surrey, UK). The urethral pressure increased significantly to levels even higher than the original urethral pressure level. In addition, we proved that the IUS and the IAS are collagen-muscle tissue cylinders by histopathological examinations.

We took tissue biopsies, and after preparation, we stained the specimens with Masson trichrome stain which stains the collagen fibers greenish-blue and the muscle fibers brown. We demonstrated lacerations in the collagen chassis of the IUS in patients with SUI, and lacerations in the collagen chassis of the IAS in patients with FI. ${ }^{3}$

The best example of CNS sympathetic training is toilet training which starts in early childhood, so does the control of body excreta "urine, flatus, and stools." Controls of "temperance" and body reactions to provocative stimuli are gained later in life through learning and experience, similar to the control of "sexual behavior" and "premature ejaculation." 1,2

Body excreta are stored and expelled through organs that develop embryological from the cloaca, the urinary bladder and the urethra in front and the rectum and the anal canal posterior.

In women, CBT causes lacerations in the strong collagen chassis of the vagina, and it is intimately related to the IUS in front, and/or the IAS posteriorly with subsequent vaginal prolapse, overactive bladder $(\mathrm{OAB})$, stress urinary incontinence (SUI), and/or FI. ${ }^{3-6}$

\section{MICTURITION AND URINARY CONTINENCE}

The urethra is a long conduit (tubular) passage to allow the flow of urine from the urinary bladder where it is stored (Figs 1 to 9). ${ }^{4-12}$ It has two sphincters to control its function, the IUS and the external urethral sphincter (EUS).

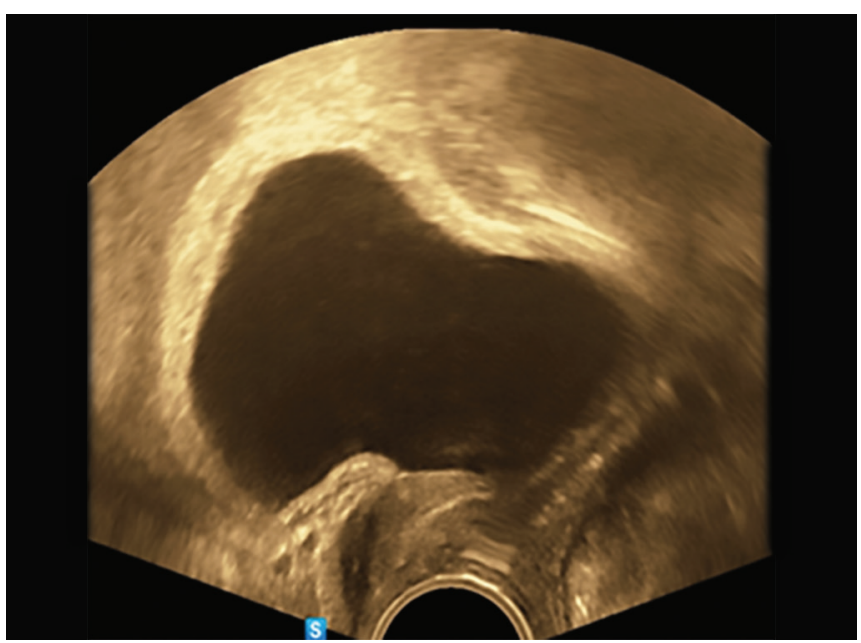

Fig. 1: Coronal section 3D US image, with normal intact IUS and IAS in a continent woman. The IUS is seen as a compact tissue cylinder with two echo seen, muscle on top of collagen. The muscle layer is continuous with the detrusor muscle. The bladder neck is closed. The IAS is a compact tissue cylinder surrounding a closed and empty anal canal
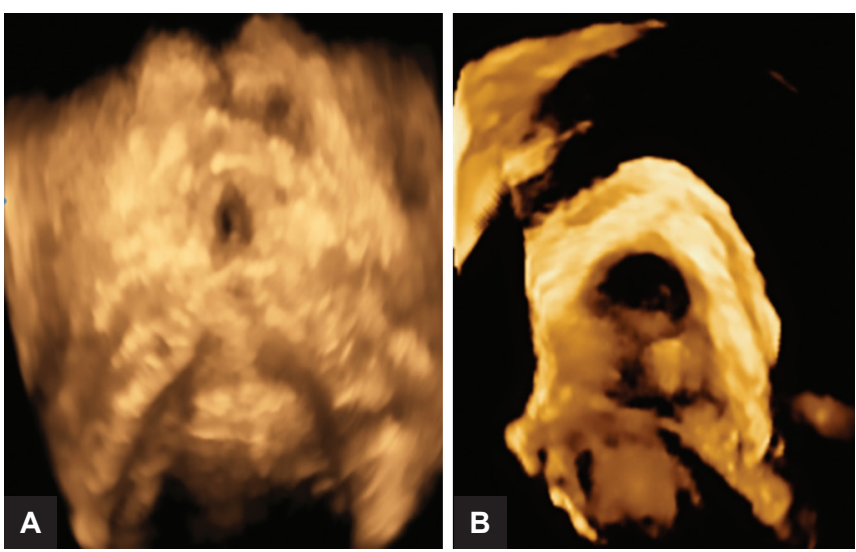

Figs 2A and B: Three-dimensional US cross-section images. (A) Normal continent woman with an intact IUS and a closed urethra and normal intact anterior vaginal wall. (B) Open urethra with lacerated posterior wall, the IUS is seen as a horse-shoe, and lacerated anterior vaginal wall with vaginal prolapse

The IUS is a collagen-muscle tissue cylinder that extends from the bladder neck down to the perineal membrane in both sexes. The muscle is plain muscle fibers that lie on and intermingle with the strong collagen sheet in the midsection. It has its nerve supply from the sympathetic nervous system (T10-L2). The EUS (compressor urethrae) is a skeletal muscle that lies in the deep perineal pouch and has its nerve supply via the pudendal nerve (S2, 3, and 4).

Before toilet training, micturition is a spinal-sacral reflex. Sensations of bladder fullness travel along the sensory nerves (S2, 3, and 4) to the sacral spinal nerve centers, where exciter impulses travel along the pelvic parasympathetic cholinergic nerves (S2, 3, and 4) causing detrusor contractions and emptying the bladder.

Toilet training initiates gaining and maintaining high sympathetic tone at the IUS, keeping the IUS contracted

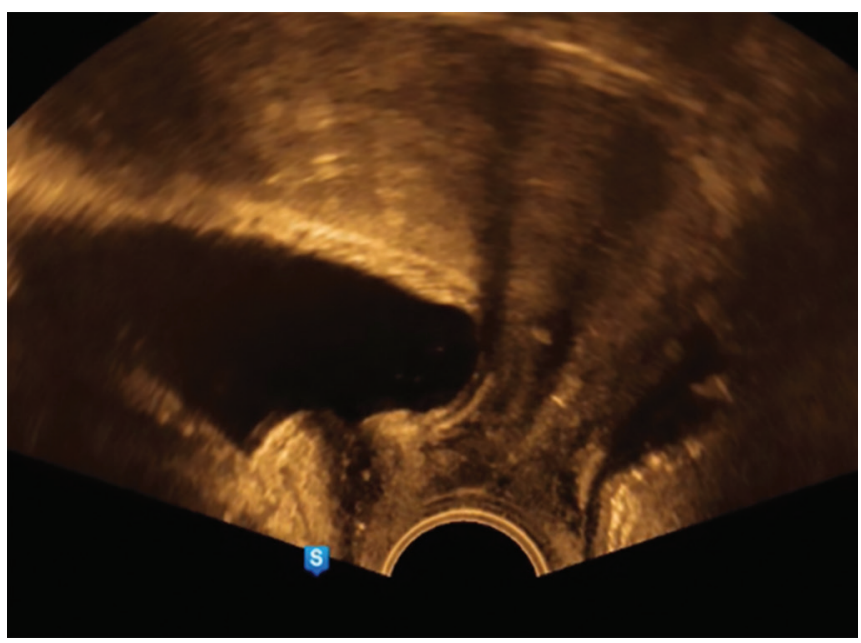

Fig. 3: Coronal section 3D US image, with normal intact IAS in fecal continent woman. The IAS is a compact tissue cylinder surrounding a closed and empty anal canal. However, the IUS is seen with torn uppermost part with an open upper part of the urethra allowing urine to enter the urethra on a sudden rise of abdominal pressure leading to $O A B$ 


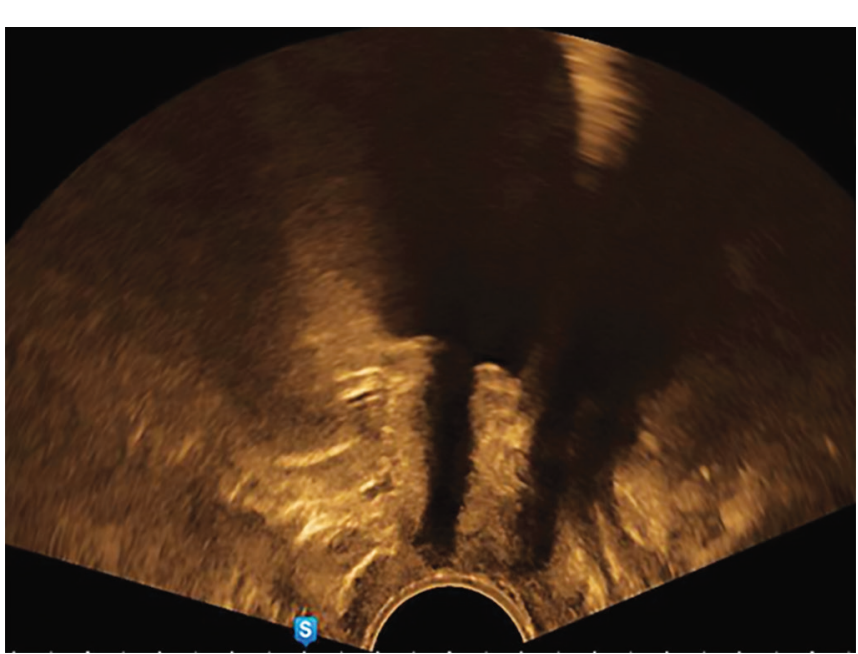

Fig. 4: Coronal section 3D US image, with torn lacerated IAS in a woman with mixed type of SUI. The whole length of IUS is torn with an open urethra allowing urine to enter the urethra on a sudden increase of abdominal pressure

and the urethra closed and empty all the time until there is a need and / or a desire to void. The trained person will, at suitable social circumstances, controlled by an alert sound CNS, inhibits the acquired high sympathetic tone at the IUS, allowing its relaxation and urine pass into the urethra. Urine entering the urethra will initiate the desire to void and through detrusor contractions, voiding occurs. The EUS will relax and urine passes undisrupted.
At the end of micturition, the EUS will contract rhythmically to squeeze the remaining urine in the urethra and leaves the urethra empty and closed again. During voiding, any sudden provocative stimulus will cause reflex increase in the sympathetic tone, which leads to contraction of the IUS interrupting the stream of urine.

Normally, the IUS is intimately lying on the anterior vaginal wall (Fig. 8). The sympathetic nerve endings produce norepinephrine as their neurotransmitter, which act on receptors on the plain muscle fibers of the IUS causing its contraction, and toilet training keeps it contracted all the time. In some children, the sympathetic nerve endings do not produce norepinephrine and so the IUS is not contracting and the urethra is not closed and empty all the time, and the open urethra will allow involuntary escape of urine. ${ }^{9}$ This will produce nocturnal enuresis (NE). It seems that in NE patients, there is overall deficiency of norepinephrine and this explains going rapidly into deep sleep on lying down. Giving ephedrine will stimulate the nerve endings to produce norepinephrine and also, ephedrine acts on the muscle receptors as alpha-sympathomimetic and causes IUS contraction and this will cure NE. ${ }^{9}$

In conclusion, urinary continence depends on intact healthy nervous system and on a closed empty urethra. The closed empty urethra depends on two factors, one
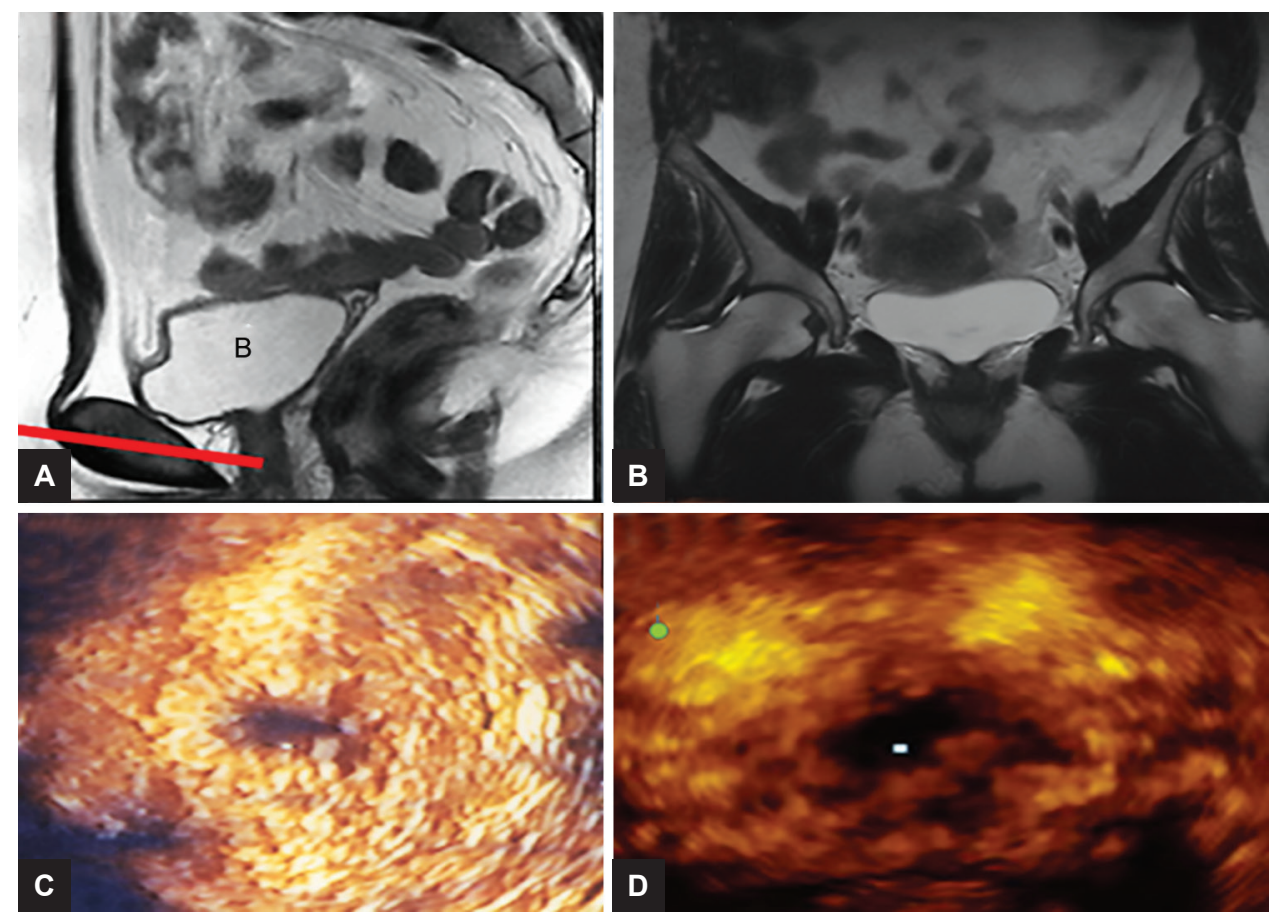

Figs 5A to D: Magnetic resonance imaging images ( $A$ and $B$ ) and cross-sections of 3D US ( $C$ and $D$ ). Image $A$ is of a patient with Mullerian duct agenesis with absent vagina and uterus, the IUS is seen as a compact tissue cylinder that extends from the bladder neck to the perineal membrane. Image $B$ is a coronal section of MRI, with torn IUS more marked in its upper part with OAB. Images $C$ and $D$ are cross-sections done by $3 D$ US, image $C$ is of healthy intact IUS seen as a cylinder of collagen with muscle fibers on top of the collagen cylinder in its mid thickness. Image $D$ is of a patient with SUI, torn IUS with an open urethra 

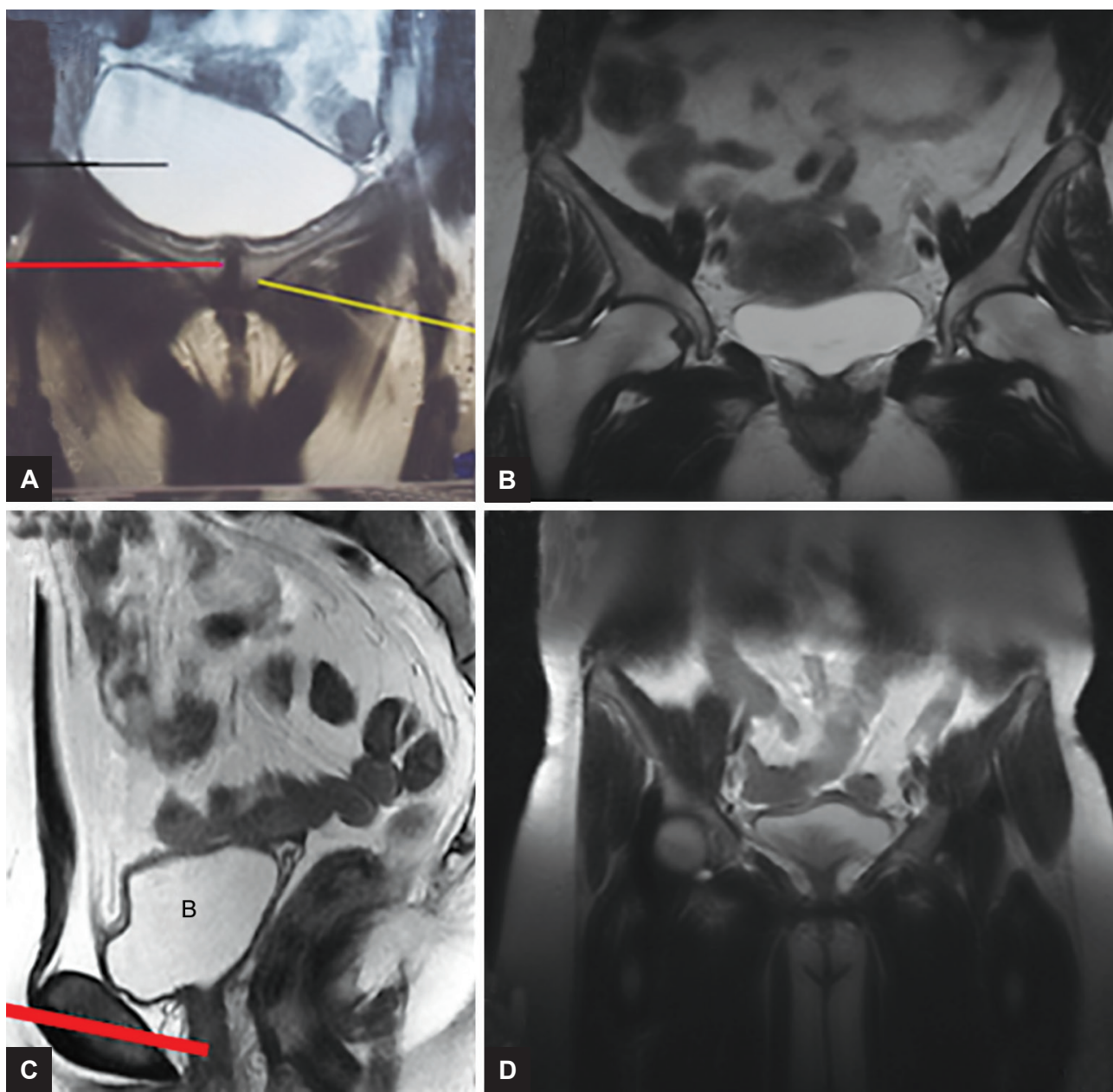

Figs 6A to D: Magnetic resonance imaging images. (A) Coronal section which shows full bladder with a closed and empty urethra. The IUS is a compact tissue cylinder that extends from the bladder neck down to the perineal membrane. (B) Sagittal section of a patient with Mullerian duct agenesis, the IUS is seen as a compact tissue cylinder that extends from the bladder neck down with a closed and empty urethra. (C and D) Coronal sections with lacerated IUS, more marked at the bladder neck causing funneling of the bladder neck leading to OAB and SUI (mixed type of SUI)

inherent and one acquired. The inherent factor is the presence of a strong intact collagen chassis with reactive muscle. The acquired factor is to keep high sympathetic tone at the IUS.

\section{DEFECATION, FECAL CONTINENCE, AND FI}

Normally in continent people, the anal canal is empty and closed all the time, with subsequent fecal continence (Figs 7, 9, and 10). ${ }^{13.14}$ The anal canal has two sphincters, the IAS, and the external anal sphincter (EAS). The IAS is a collagen-muscle tissue cylinder that surrounds the anal canal. The muscle is plain muscle fibers that lie on and intermingle with the collagen sheet in its middle area. It has its exciter nerve supply from the sympathetic nerves (T10-L2). The IAS surrounds the anal canal and is surrounded in its lower part by the EAS. The EAS is a skeletal voluntary muscle innervated via the pudendal nerve, and is divided into three parts, subcutaneous, superficial, and deep parts. Toilet training
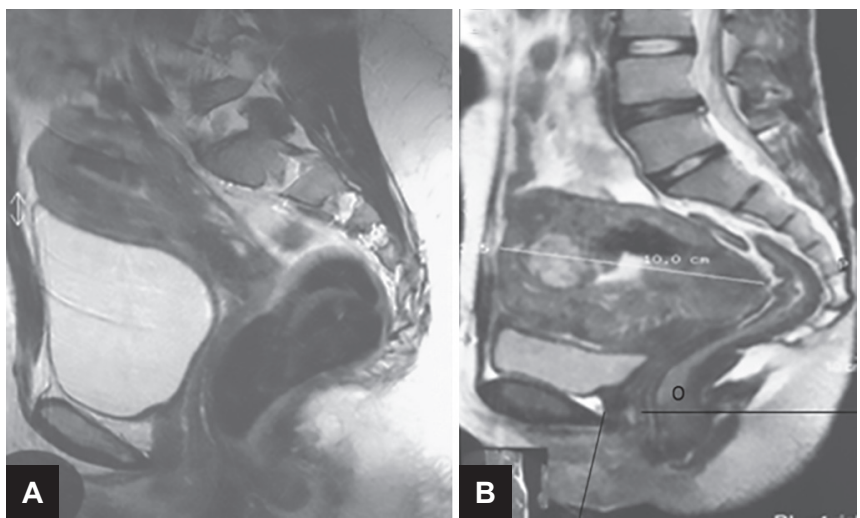

Figs 7A and B: Magnetic resonance imaging images. (A) Normal continent woman with full bladder and an intact IUS is seen as a compact tissue cylinder that extends from the bladder neck to the perineal membrane. Although the rectum is full, an intact IAS is keeping fecal continence. (B) Sagittal section of MRI of a patient who has urinary continence with an intact IUS, and FI with lacerated IAS and an open anal canal. The patient has fibroid uterus which causes increased abdominal pressure, but still the patient has a normal IUS which is seen extending from the bladder neck to the perineal membrane. $V$ is the vagina and kindly notice how thick is the IUS compared with nonprolapsed vagina 


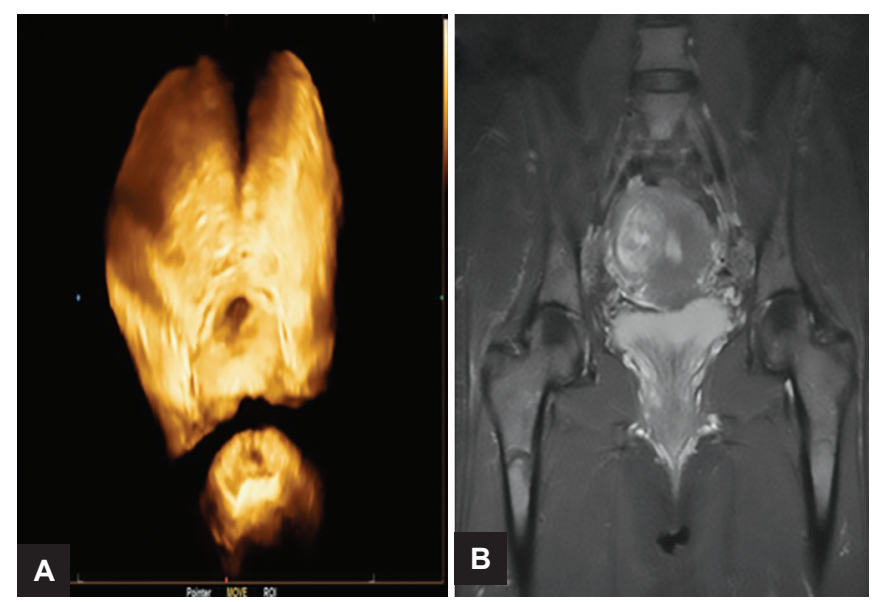

Figs 8A and B: (A) Cross-section of 3D US image of a lacerated IUS of a patient with SUI. (B) Coronal section of MRI image showing lacerated IUS with funneling of the bladder neck and an open urethra of a patient with OAB and SUI (mixed SUI)

leads to gaining and maintaining high sympathetic tone at the IAS that keeps it contracted and the anal canal empty and closed all the time. At the anorectal region, there are nerve sensors for sensations like distension, texture, and nature of the contents, temperature, touch, and friction.

Before toilet training, distension of anorectal area will induce rectal contractions, increase of the abdominal pressure (through contractions of the diaphragm and abdominal muscles), pushing the rectal contents through an open anal canal and relaxed EAS.

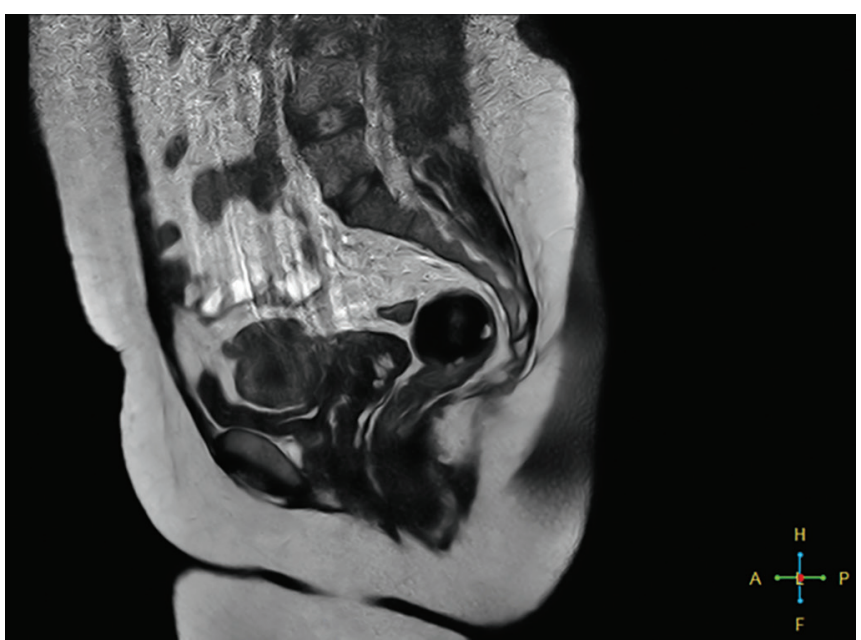

Fig. 9: Magnetic resonance imaging sagittal section image of a patient with SUI, vaginal prolapse, and FI. The IUS is lacerated, the vagina is seen lax, and the IAS is seen lacerated with an open anal canal

Toilet training initiates gaining and keeping high sympathetic tone at the IAS, keeping it contracted and the anal canal empty and closed all the time until there is a desire and/or a need to pass flatus and/or feces at suitable social circumstances. Suitable social circumstances allow the person to inhibit the acquired high sympathetic tone at the IAS for a moment to pass flatus or for a longer time to pass stools. The EAS is a voluntary muscle controlled by the systemic nervous system, (through the pudendal nerve), mastered by healthy alert CNS. In women, the IAS is lying in close relation with the posterior vaginal wall (Fig. 9).
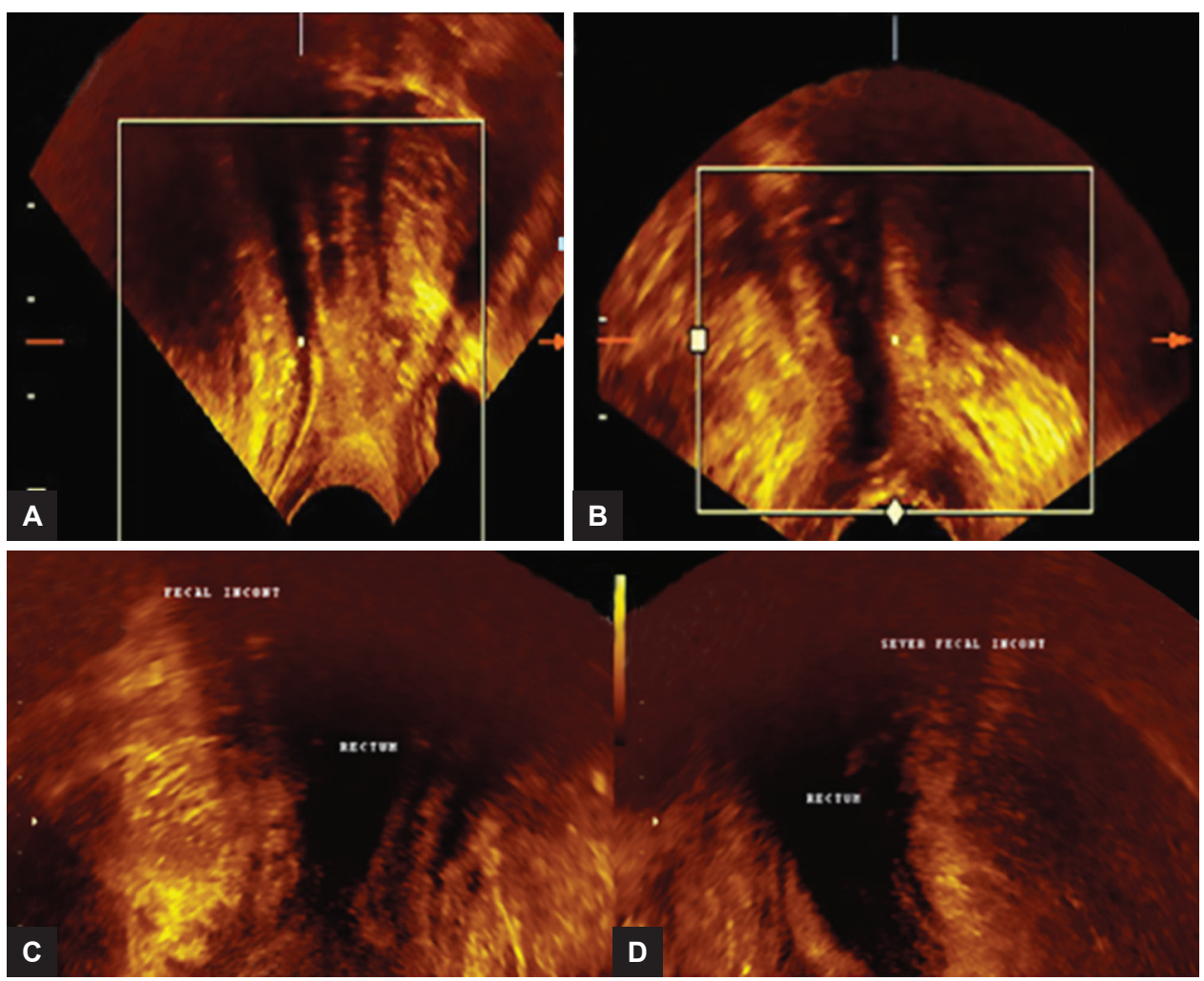

Figs 10A to D: Three-dimensional US images. (A) Normal intact IAS, with closed empty anal canal. (B-D) Open anal canal with torn IAS in patients with FI 


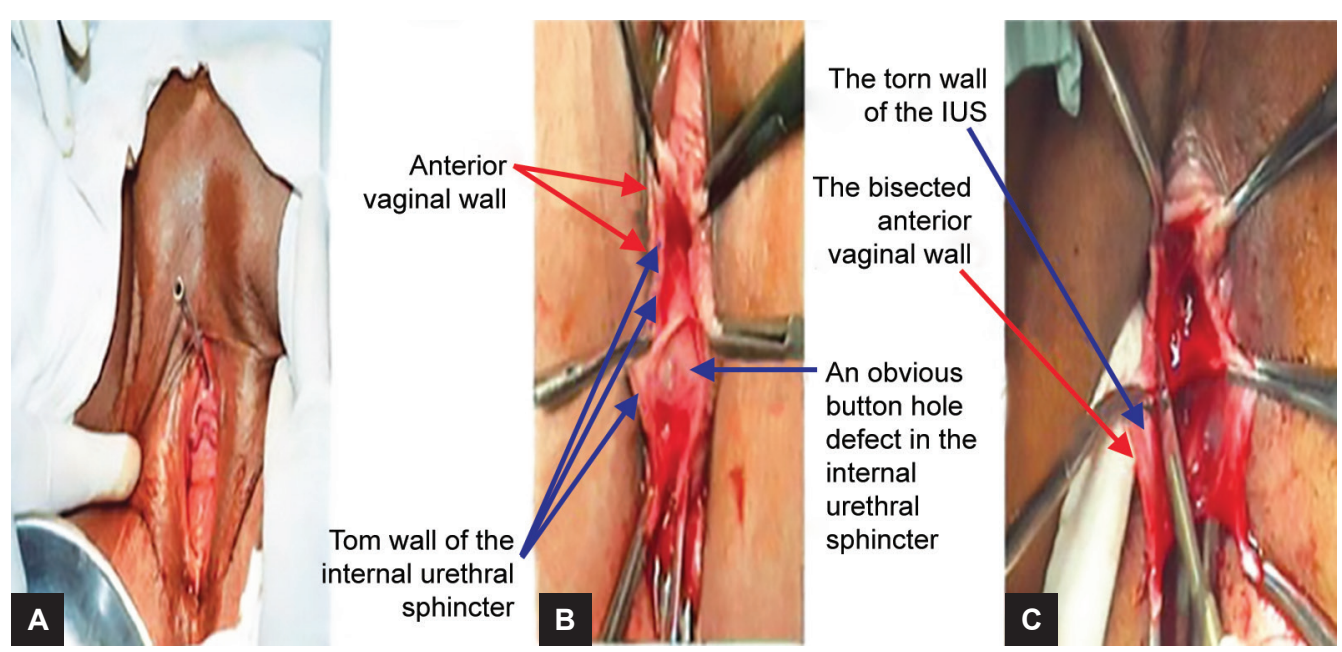

Figs 11A to $C$ : $(A)$ The anterior and the posterior vaginal walls are seen without straining, which means vaginal wall prolapse. A metal catheter is pointing upward and anterior, which means loss of posterior $\mathrm{U}-\mathrm{V}$ angle. (B and C) Lacerated IUS and two edges in the vaginal flap, one belongs to the IUS and the other edge to the anterior vaginal wall

\section{THE VAGINA}

The vagina is a collagen-elastic-muscular tube that extends from the vulva in an upward and backward direction for about 4 to 5 inches, with the uterus at the top and opens on the anterior vaginal wall near its vault (Figs 7, 9, and 11). It is held in its upward-backward direction by the strong pelvic ligaments and its firm collagen chassis. The tough strong pelvic ligaments (the uterosacral, the great transverse ligaments and the pubocervical ligaments) attach the uterine cervix and the upper part of the vagina to the sacrum and the pelvic walls.

The CBT causes lacerations in the strong collagen chassis of the vagina, affecting mostly its transverse axis, leading to wide patulous vagina and redundant vaginal walls with subsequent vaginal prolapse. The intimate structures, the IUS lying on the anterior vaginal wall, and/or the IAS lying on the posterior vaginal wall will have their tough collagen chassis lacerated as well. This will produce a torn weak IUS which cannot stand against sudden rise of abdominal pressure and urine will leak. When the woman feels wet, reflex general sympathetic activity is induced which will increase the existing sympathetic tone at the IUS closing the urethra, which prevents further leak of urine (SUI).

The IAS, which is intimately lying on the posterior vaginal wall, will suffer from damage and lacerations of its collagen chassis with the posterior vaginal wall with subsequent $\mathrm{FI}$ and posterior vaginal wall prolapse.

The OAB and SUI are not pathognomonic to women; men can suffer from OAB and SUI. Conditions which lead to opening of the uppermost part of the urethra, like in prostatic hyperplasia with nonsymmetrical compression on the upper part of the urethra, allow the passage of urine to the open uppermost part of the urethra. This will initiate an urgent desire to void, urgency, which may lead to urgency incontinence, OAB, and SUI.

\section{THE UTERUS AND VAGINAL VAULT}

The pelvic ligaments (the uterosacral, the great transverse cervical, and the pubocervical ligaments) fix the uterine cervix and the upper part of the vagina to pelvic walls and the sacrum. Uterine and vault prolapse are due to weakness and laxity of the pelvic ligaments.

\section{Diagnosis of Genital Prolapse and Body Excreta Incontinence}

Diagnosis of structural damage can be achieved clinically and by medical imaging [X-ray CT scan, US, especially threedimensional (3D) or four-dimensional US and MRI]. ${ }^{3-6,10,12}$

\section{Clinical, Medical History, and Examination}

The close relation of the IUS and the IAS with the vagina is the main factor of outlet control incontinence in cases of vaginal prolapse.

\section{Symptoms of Vaginal Prolapse}

- Vaginal pressure, "something coming down," a protruding mass at first on straining and vaginal discharge

- Symptoms are aggravated by vigorous activity, prolonged standing, coughing, sneezing, or straining

- Relief can be obtained by rest, or lying down.

- Urinary symptoms, e.g., frequency, double voiding, urgency, dysuria and incontinence, SUI

- Fecal incontinence

\section{Signs of Genital Prolapse}

- Relaxed vaginal outlet, and enlarged genital hiatus

- Short perineum 
- Mass - the examiner must notice its nature, site, size, and any ulceration

- The genital prolapse increases on straining

- The examiner must ask and look for the presence of SUI and FI

We added two important signs to be mentioned ${ }^{18}$ :

1. The external urethral meatus is normally directed forward; if it is directed upward and forward, this means loss of posterior urethrovesical angle.

2. Normally you cannot see the anterior or the posterior vaginal walls at the introitus, as they are going upward and backward; if you can see one or both of them without asking the patient to strain, this means vaginal prolapse.

\section{INVESTIGATIONS}

Structural damage is diagnosed by medical imaging. Functional disturbances need laboratory investigations, e.g., urine analysis; catheter to measure the volume of residual urine, culture and sensitivity test; and urodynamic study.

Lacerations of the collagen chassis of the IUS, IAS, and the vagina are diagnosed by medical imaging, e.g., US and MRI, US imaging using 3D transvaginal probe of Voluson 730 pro- (GE machine) with multifrequency 4 to $8 \mathrm{MHz}$ (using an average $6.5 \mathrm{MHz}$ intracavity probe). With a semifull bladder, the patients were scanned in the spine position with flexed legs. The transvaginal volumetric probe was put in the lower part of the vagina toward the urethra anterior and to the anal canal and the IAS posterior. Multiplane 3D sections were taken.

Magnetic resonance imaging-MR machine (Philips, $1.5 \mathrm{~T}$ ) with multiplane reconstruction. MRI Image Acquisition-Patients were imaged in MRI unit (1.5 T, Philips ${ }^{\circledR}$ ) sagittal and coronal T2, using the following parameters: TR (repetition time) $=4000$, TE (echo time) $=102$, Matrix $320 \times 320$ for sagittal and $384 \times 320$ for coronal; field of view $30 \mathrm{~cm}$ for sagittal and $38 \mathrm{~cm}$ for coronal; section thickness $3 \mathrm{~mm}$ for sagittal and $5 \mathrm{~mm}$ for coronal.

Structural damage leads to functional abnormality. Damage and lacerations of the collagen chassis of any organ alter its function, and this is well demonstrated in urinary and fecal continence/incontinence. Lacerations of the collagen chassis of the IUS and/or the IAS lead to weak sphincters which are unable to stand against a sudden rise of abdominal pressure and incontinence ensues.

\section{Reconstructive Surgery for Pelvic Floor Dysfunction}

Mending the torn collagen chassis of the IUS restores its ability to resist sudden increases of abdominal pressure (Figs 11 and 12). ${ }^{13-18}$ Adding extra support will help, so if we do overlapping of the two longitudinal anterior vaginal wall flaps, we add additional strength to the
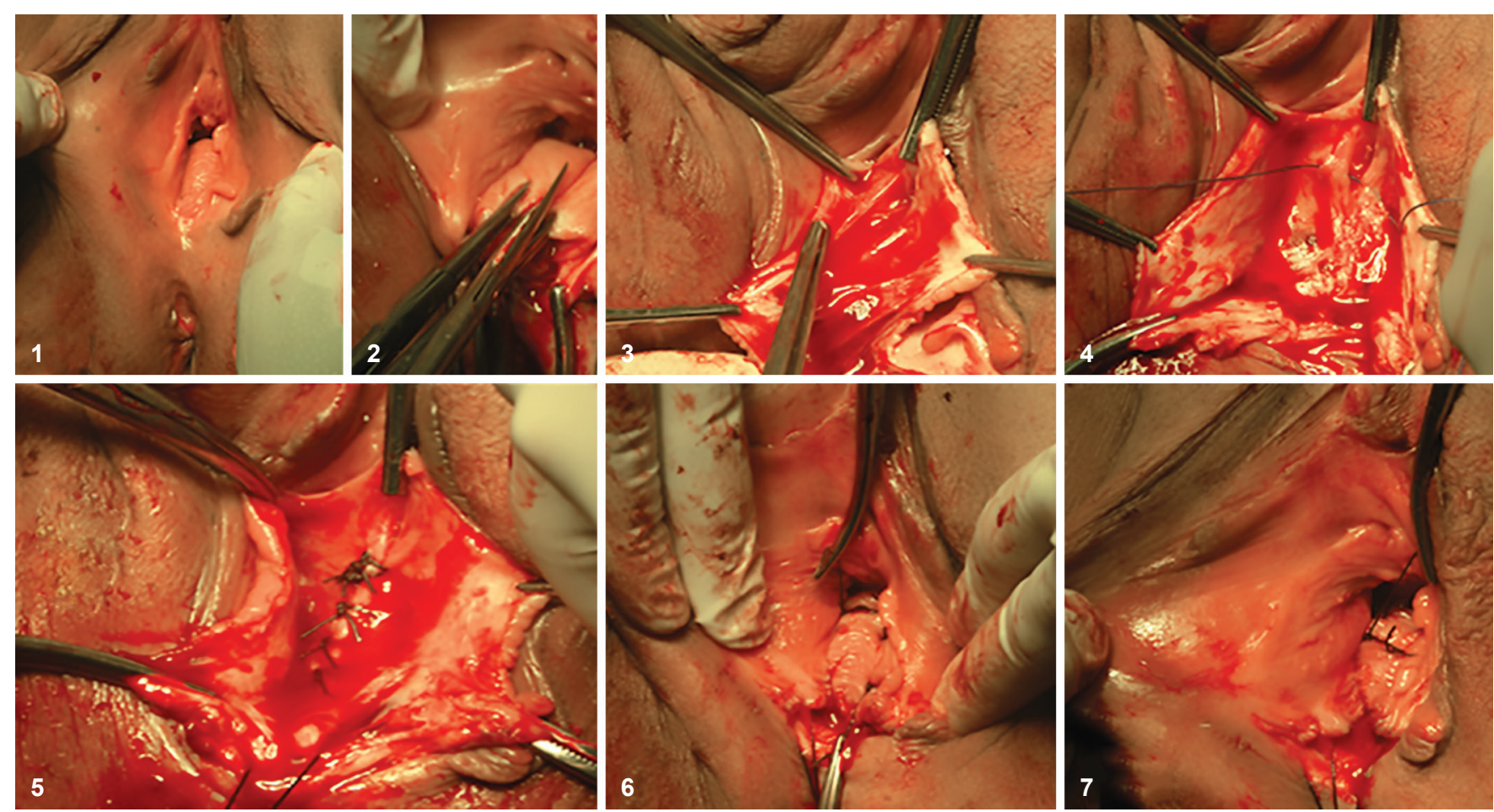

Fig. 12: In the theater, steps of posterior repair, image 1 shows posterior vaginal wall prolapse as a bulge, cut in image 2 . Image 3 shows the defect in the IAS as being dissected from the posterior vaginal wall. Figure 4 shows the start to mend the IAS and is seen complete in image 5. Images 6 and 7 show overlapping of the two vaginal flaps and the two levators are approximated by two surgical sutures not tied and the thread is held on artery forceps to be tied after finishing the vaginal overlap. Then repair of the perineum is done 
mended IUS, and we strengthen the lacerated lax prolapsed anterior vaginal wall. The same concept applies to the IAS and the posterior vaginal wall. Applying this concept, we introduced a vaginal reconstructive operation "urethro-ano-vaginoplasty" to correct vaginal prolapse and body excreta incontinence.

The operation consists of an anterior part and a posterior part and can be done under general or spinal anesthesia.

The anterior part, visualizing the uterine cervix through vaginal speculum, holds the cervix by two pairs of vulsellum. Then, we inject (10-30 mL) subvaginal, normal saline with 2/1,000 adrenaline to help as hydrodissection, between the anterior vaginal wall and the IUS; and help in hemostasis. We cut a transverse incision in the anterior vaginal wall about one inch long and one inch above the external cervical os. Using a pair of dissecting scissors, we separate the anterior vaginal wall from the IUS; then cut the anterior vaginal wall longitudinally up to the submeatal sulcus. Holding each vaginal flap with three pairs of Kocher forceps, we can recognize the adherent wall of the IUS and dissect it free from the vaginal flap. When this is done on both sides, we will see the laceration in the collagen chassis of the IUS, mend it, with fine slowly absorbed, simple interrupted 5 to 6 stitches. We add extra support to the mended IUS by overlapping the two vaginal flaps.

In the posterior part, we correct the FI and the lacerated prolapsed posterior vaginal wall. We use hydrodissection as done anteriorly. We expose the torn collagen chassis of the IAS by separating the torn collagen chassis of the IAS clear from the posterior vaginal wall, and the lacerations in the anterior wall of the IAS will appear. Start mending the torn collagen chassis of the IAS using slowly absorbed suture material (Vicryl number one zero) by simple interrupted stitches; this may take 4 to 6 simple stitches. Approximate the two levator ani muscles, using number one slowly absorbed suture material, leave the thread on pairs of artery forceps untied until later after overlapping the two longitudinal flaps of the posterior vaginal wall; two stitches to approximate the levators may be enough. Strengthen the posterior vaginal wall by overlapping the two longitudinal vaginal flaps; also, we add extra support to the mended IAS, correct the posterior vaginal wall prolapse, and narrow the patulous vagina. We approximate the two levator ani muscles by tying the threads by surgical knots. Finish by repairing the perineum.

\section{DISCUSSION}

Although the sympathetic nervous system is a part of the autonomic nervous system, which is described to act automatically without voluntary control, yet we can train it from early childhood and all through life. ${ }^{17,18}$ The best example is toilet training, the mother starts to train her child as early as about 2 years of age, how to hold himself till proper social circumstances are available. This is gained by learning how to acquire and maintain high sympathetic tone at the IUS all the time, thus keeping the urethra empty and closed all the time until there is a need and/or a desire to void and suitable social circumstances allow. The presence of urine in the urethra initiates the desire to void. Torn uppermost part of the IUS will, on rise of abdominal pressure, allow urine to enter the open upper part of the urethra and cause urgent desire to void (urge and urgency incontinence and $\mathrm{OAB}$ ). Childbirth trauma is the most frequent cause of lacerations and weakness of the IUS. Other causes like estrogen deficiency, getting older after menopause, and chronic or repeated genitourinary infections lead to weakness of the pelvic collagen, and/or congenital collagen weakness can start or aggravate the condition. Other important example of learning and training the sympathetic system is that you can train the domestic animal to evacuate the cloaca at the site you trained them to do so.

In men, prostatic hyperplasia causes uneven pressure on the upper part of the urethra leading to irregular dilatation of the upper most part of the urethra allowing urine to enter the urethra causing a sudden desire to void (urge and OAB), increased frequency, nocturia, urgency, and incontinence. In the toilet, it may be difficult to start voiding, because the man has to inhibit the acquired high sympathetic tone at the IUS and meanwhile has to open the urethra which is compressed by the enlarged prostate, (hesitancy). Any annoying stimuli will increase the sympathetic tone and thus contract the IUS that leads to an interrupted stream and feeling of incomplete emptying of the bladder.

Mainly, the same mechanism of voiding and UI applies to the control of defecation and FI; but here it is more complex as the IAS is responsible for holding flatus and stools whether liquid or solid. An alert healthy CNS will supervise, control, and coordinate the actions to be taken governed by social circumstances available.

Growing up and getting wiser, and by acquitting more education and culture, by gaining tolerance and forgiveness, good faith and good conscience attitude, and by gaining more experience and training, the person will learn, controlled by an alert healthy mind, how to control verbal, physical, and sexual behavior induced by various provocative stimuli. This is gained through the control of the mind and the sympathetic nervous system supervised by an alert healthy CNS. Loss of high CNS control can be temporary or permanent.

Temporary loss of the high CNS control occurs in situations like fear and getting drunk, which lead to failure of outlet control and incontinence. Permanent failure of the 
control can occur in nervous system damage as in stroke and CNS damage by diseases like multiple sclerosis and systemic lupus erythematosus. Also, facultative damage like in Alzheimer's conditions can cause loss of outlet control and incontinence.

Medical imaging with MRI and 3D US is very efficient in showing the lacerations in the IUS and the IAS, and the vagina US is much easier, cheaper than MRI, and the recordings can be reevaluated on getting it back for further study.

\section{REFERENCES}

1. Abdel KMEH, Laila AMM, Ahmad ME. Outlet control. J Gynecol Women's Health 2017;3(3):555-620.

2. El Hemaly AKM, Mousa LA, Kandil IM, Hussein KS. Continence. Reprod Syst Sex Disord 2016;5:186.

3. El Hemaly AKMA, Mousa LAE. Stress urinary incontinence, a new concept. Eur J Obstet Gynecol Reprod Biol 1996; 68:129-135.

4. El Hemaly AKMA, Kandil IM, Kurjak A, Mousa LAS, Kamel HH, Serour AG. Ultrasonic assessment of the urethra and the vagina in normal continent women and women suffering from stress urinary incontinence and vaginal prolapse. Donald School J Ultrasound Obstet Gynecol 2011 Oct-Dec;5(4):330-338.

5. El Hemaly AKMA, Kandil IM, Kurjak A, Serour AG, Mousa LAS, Zaied AM, El Sheikha KZ. Imaging the internal urethral sphincter and the vagina in normal women and women suffering from stress urinary incontinence and vaginal prolapse. Gynaecol Perinatol 2009 Oct-Dec;18(4):169-286.

6. El Hemaly AKMA, Kandil IM, Kurjak A, Mousa LAS, Kamel HH, Serour AG. Ultrasound assessment of the internal anal sphincter in women with fecal incontinence and posterior vaginal wall prolapse (rectocele). Donald School J Ultrasound Obstet Gynecol 2011 Oct-Dec;5(4):330-342.

7. El Hemaly AKMA, Mousa LAS, Kandil IM, El Sokkary FS, Serour AG, Hussein H. Fecal incontinence, a novel concept: the role of the internal anal sphincter (IAS) in defecation and fecal incontinence. Gynaecol Perinatol 2010 Apr-Jun;19(2):79-85.
8. El Hemaly AKMA, Mousa LAS. Micturition and urinary continence. Int J Gynecol Obstet 1996;42:291-292.

9. El Hemaly AKMA. Nocturnal enuresis: pathogenesis and treatment. Int Urogynecol J Pelvic Floor Dysfunct 1998; 9:129-131.

10. El Hemaly AKMA, Mousa LAES, Kandil IM, Shehata KA. Imaging of the pelvic floor. Curr Med Imag Rev 2014;10(3): 205-214.

11. El Hemaly AKM, Mousa LA, Kandil IM. The concept and pathophysiology of urinary incontinence. In: Alhasso A, Fernando A, editors. Urinary incontinence. INTECH Publication; April 2012. pp. 145-160.

12. Kandil IM, El Hemaly AKM, Radwan MM: Ultrasonic assessment of the internal urethral sphincter in stress urinary incontinence. Int J Gynecol Obstet 2003;2(1).

13. El Hemaly AKM, Mousa LA, and Kandil IM, Al Sayed MS, Zaher MA, Soliman MSA, Serour AG. A novel concept on the patho-physiology of defecation and fecal incontinence (FI) in women-moreover, its reconstructive surgery. In: Catto-Smith AG, editor. Fecal incontinence causes, management and outcome. INTECH Publication; April 2014. pp. 47-67.

14. El Hemaly AKM, Mousa LA, Kurjak A, Kandil IM, Serour AG. Pelvic floor dysfunction, the role of imaging and reconstructive surgery. Donald School J Ultrasound Obstet Gynecol 2013 Jan-Mar;7(1):86-97.

15. El Hemaly AKM, Mousa LAS, Kandil IM, El Sokkary FS, Serour AG, Hussein H. Surgical treatment of stress urinary incontinence, fecal incontinence and vaginal prolapse by a novel operation "urethro-ano-vaginoplasty". Gynaecol Perinatol 2010 Jul-Sep;19(3):129-188.

16. El Hemaly AKM, Mousa LAES, Kandil IM, Al-Adwani AKA. Pelvic floor dysfunction and its reconstructive surgery: novel concepts; Createspace, an ISBN 978-1-001-04115, Amazon Company; 2014.

17. El Hemaly AKM, Mousa L, Kandil IM. Continence and Incontinence: How Can You Gain Continence? ISBN 978-3639-66683-0, Scholars press. November 2014.

18. El Hemaly AKM, Mousa LAES, Kandil IM; Pelvic floor dysfunction: new concepts on its patho physiology and its reconstructive surgery. Gynecol Obstet Case Report 2016 Jan. 